\title{
Regionale Bezüge der deutschsprachigen Wissensvermittlung im baltischen Raum
}

\author{
Linguistische Beobachtungen am Beispiel livländischer \\ Quellen des 17. Jahrhunderts
}

\section{Vorbemerkungen}

Die ältesten wissenschaftlichen und populärwissenschaftlichen deutschsprachigen Texte aus dem Baltikum ${ }^{1}$ haben traditionell zum Interessenbereich der Wissenschaftshistoriker gehört (vgl. Stradiņ̌̌ 2009). Abgesehen von vereinzelten Untersuchungen, vorwiegend zum Lautbestand und zu lexikalischen Lehnprozessen, sind diese Fachtexte kaum von der Linguistik beachtet worden. Texte, die im Baltikum entstanden sind, haben eine bedeutende Rolle für die Wissensvermittlung in der Region sowie auch über die regionalen Grenzen hinaus gespielt und verdienen schon aus diesem Grunde gebührende Aufmerksamkeit auch aus der linguistischen Perspektive. Dies betrifft auch die für den vorliegenden Beitrag gewählten zwei Publikationen aus dem 17. Jahrhundert Stratagema oeconomicum ${ }^{2}$ von Salomon Gubert und Lieffländifcher Landman von Johann Hermann von Neidenburg. ${ }^{3}$ Beide Texte sind in der gegenwärtigen Forschung nicht nur als historische Denkmäler der Agrargeschichte von Interesse. Im Stratagema oeconomicum von Gubert geht es neben der livländischen Bodenkunde und Landwirtschaft auch um astronomisches Wissen, um ethnographische Gegebenheiten und Haushaltsführung und es könnte auch For-

1 Der Begriff Baltikum wird hier auf Grund der gemeinsamen Geschichte auf die gegenwärtigen Staaten Estland und Lettland - die Nachfolgeterritorien des ehemaligen Altlivlands - eingeschränkt. Mit Livland (hist. auch Lieffland) ist das sog. Schwedisch-Livland gemeint - das nördlich der Daugava (hist. Düna) und südwestlich des Peipussees unter schwedischer Oberherrschaft 1629-1721 von Letten und Esten besiedelte und deutschsprachig verwaltete Territorium.

2 Hier und im Weiteren wird die Abkürzung des Titels als Stratagema oeconomicum verwendet, den vollen Titel s. im Literaturverzeichnis.

3 Im Weiteren wird der Name Johann Hermann gebraucht, wie er in den deutschbaltischen biographischen Werken angeführt ist (vgl. Gadebusch 1777, II: 65; Recke \& Napiersky 1829: 259).

Dzintra Lele-Rozentāle, Fakultät für Übersetzen und Dolmetschen, Hochschule Ventspils, Inženieru 101, LV-3601, Ventspils, Lettland, E-Mail: dzintra.lele-rozentale@venta.lv

○ Open Access. (c) 2018 Dzintra Lele-Rozentāle, publiziert von De Gruyter. (cc))BY-NC-ND Dieses Werk ist lizenziert unter der Creative Commons Attribution-NonCommercial-NoDerivatives 4.0 Lizenz.

https://doi.org/10.1515/9783110476958-010 
scher der Volkskunde, Kulturgeschichte und Medizin interessieren (vgl. dazu Stradiņš 2009: 160). Hermann beschreibt in erster Linie die landwirtschaftlichen Arbeiten und in Verbindung damit auch die livländische Bodenbeschaffenheit. Außerdem bietet er wertvolles Material für sozialhistorische Untersuchungen, z. B. zum Thema Güterverwaltung (vgl. den Beitrag von Turzyński 2011). Beide Texte sind z. T. in der herkömmlichen Tradition der deutschsprachigen Handbücher für Landwirte geschrieben, sie sind aber an die livländischen Verhältnisse angepasst. Als Nachweis ihrer praktischen Anwendbarkeit gelten wiederholte Auflagen und spätere Übersetzungen ins Russische und Polnische. Eine aus der linguistischen Sicht wichtige Frage, auf die im Weiteren auch eingegangen wird, lautet: Wie vollzieht sich die Anpassung der wissensvermittelnde Texte an die regionalen Gegebenheiten? Daraus entsteht eine weitere Frage, und zwar: Lassen sich beide Texte als Repräsentanten der regionalen deutschen Varietät einstufen? Die Beantwortung dieser Fragen sieht die Beschäftigung mit der Adressatenbezogenheit vor, die in Struktur und Themenwahl, in den gewählten sprachlichen Ausdrucksformen und auch in der Verfachsprachlichung der Inhalte vorausgesetzt werden kann. Auf Grund des bisherigen Forschungsstandes kann davon ausgegangen werden, dass beide Texte Züge der regionalen Varietät des Deutschen - des sog. baltischen Deutsch - aufweisen.

\section{Verfasser, Quellen und deren Charakteristik}

Das 1645 gedruckte Landwirtschaftsbuch Stratagema oeconomicum, das wohl bekannteste livländische Handbuch des 17. Jahrhunderts, stammt von Salomon Gubert (um 1590-1653) - Pastor zu Sonfel (dt. sonst Sunzel, heute lett. Suntaži), der mit Recht als „Vater der livländischen Landbauwissenschaft“ bezeichnet wird (vgl. Amelung 1885: 147). Zur Zeit des Erscheinens seines Werkes im Druck konnte Gubert auf vierzigjährige Erfahrung zurückblicken (Amelung 1884: 709-710). Sein im Folgenden mehrfach aufgelegtes Handbuch ist seit der zweiten Hälfte des 19. Jahrhunderts vielfach im Rahmen der Geschichts- und Sozialwissenschaften, der Agrargeschichte und Bodenkunde (vgl. u.a. Amelung 1885, Reintam et al. 2005, Stradiņš 2009) sowie auch in der germanistischen Fachliteratur - vorwiegend aus kontaktlinguistischer Perspektive behandelt worden (vgl. Uustalu 1982, 1984, 1985, 1990). Die dem Handbuch angehängten lettischen geistlichen Lieder sind auch in der lettischen Sprachwissenschaft analysiert worden (Andronova 2006).

Die beiden ersten Auflagen von Guberts Stratagema oeconomicum (1645 und 1649) wurden in Riga bei Schröder gedruckt. Die zweite Auflage erhielt 
auf dem Titelblatt eine Ergänzung - Itzo zum andern mahl mit fleiß über fehen/ vnd an vielen örtern vermeret vnd verbeffert. Die weiteren Auflagen erfolgten 1673, 1688 und 1757, was aber in der älteren Fachliteratur oft fehlerhaft dargestellt ist. Recke \& Napiersky (1829: 40) erwähnen insgesamt vier Auflagen die von 1645 und 1649 bei Schröder, die dritte 1688 bei Nöller und als vierte die 1757 bei Frölich veröffentlichte Ausgabe. Die Letzte enthält auf dem Titelblatt die Bemerkung des Druckers Anjetzo nach der andern verbefferten und bewährt befundenen Auflage, auf vielfältiges Nachfragen zum drittenmahle gedruckt, d. h. zwei Auflagen - die von 1673 und 1688 - sind hier gar nicht mitgezählt. Der Eintrag zu Gubert bei Gadebusch (1777, I: 456) enthält nur vier Jahreszahlen - 1645, 1649, 1688 und 1757. Die Ausgabe von 1688 ist Nun auffs neue überfehen und mit nützlichen Regiftern verbeffert. Die im vorliegenden Aufsatz gewählte Auflage von 1673, gedruckt bei Bessemesser, ist eigentlich die Dritte in dieser Reihe, die, abgesehen vom einleitenden Teil, mit derjenigen von 1649 übereinstimmt. Die Differenzen in den Jahresangaben haben sich wahrscheinlich im Laufe des 18. Jahrhunderts eingeschlichen und sind in der Folgezeit immer wieder übernommen worden, manchmal auch fehlerhaft, wie z. B. bei Adelung (1787: 1647), wo als Erscheinungsjahre 1645, 1649, 1688 und 1765 (!) angeführt sind. Bei Amelung finden sich Angaben zu sechs Auflagen: 1645, 1648 (!), 1668 (!), 1673, 1688, 1757 (1884: 721, Anm. 1). In der gegenwärtigen Forschung werden verschiedene Auflagen mit Ausnahme der Erstausgabe herangezogen, da sie in Bezug auf den Haupttext kaum Unterschiede aufweisen. Die hier gewählte Auflage, die im Erscheinungsjahr derjenigen von Hermanns Werk am nächsten steht, stimmt mit der von Gubert selbst vermehrten Auflage von 1649 überein, die festgestellten, vereinzelt auftretenden Differenzen sind vorwiegend drucktechnisch bedingt.

In der Vorrede zu seinem Tractätlein, wie Gubert es selbst im Register und in der Vorrede bezeichnet, wendet sich der Verfasser an den Leser, indem er seine für einen Pastor ungewöhnliche Beschäftigung zu rechtfertigen versucht:

Ich muß jährlich predigen: Vom Weinberge: viererlei Acker: vom Brod: Gluckhenne: vom guten Hirten: vom verlornen Schafe: von Fifcherey: vom ungerechten Haußhalter: c. von Auffätzigen: Wafferfüchtigen: Gichtbrüchtigen: und pro re nata auß dem Naturbuch Gleichniffe einführen. Wil kürze halben nicht gedencken/ wie uns die Schrifft in das Naturbuch führet. [...] Die anderen haben haben das Wörtlein Stratagema ${ }^{4}$ unfreundlich gehechelt: Die weife ich in das Alftedii Stratagematographiam in Enciiclopaed. pag. 2395. der wird fie beffer urtheilen lehren. (Gubert 1673: Vorrede)

4 Die hier und im Weiteren in Zitaten kursiv markierten Fremdwörter, lateinische Zitatwörter und zitierte Passagen sind im Original in Antiqua gesetzt, im Text integrierte anderssprachige Beispiele werden dagegen recte angeführt. 
Die wiederholten Auflagen zeugen von einer ständigen Nachfrage. Als eventuelle Adressaten nennt Amelung (1884: 722) die gebildeten livländischen Landwirte, Gutsbesitzer und -verwalter:

Der Ackerstudent Guberts war in den Händen eines jeden gebildeten Landwirths in Livland und Hunderte der häufig nicht selbst wirthschaftenden Gutsbesitzer, vorzüglich aber die zahlreichen bürgerlichen Gutsverwalter bedienten sich desselben als eines in seiner Art einzigen und unentbehrlichen Lehrmittels.

Güntz (1897: 9) zählt Gubert neben Coler, Grosser, Höfler, Picus, Grützmann und Germershausen zur Gruppe der landwirtschaftlichen Schriftsteller, die aus protestantischen Pfarrern bestand. Gubert hat in sein Buch auch die unveröffentlichten Materialien des Astronomen, Astrologen und Mediziners Zacharias Stopius oder Stoppe (um 1535-Ende des 16./Anf. des 17. Jhs.) aufgenommen, worauf im Text (Gubert 1673: 45) auch ein expliziter Hinweis zu finden ist, vgl. Das III. Capitel. Von der Sonnen Auf= und Niedergang/ Tag= und Nachtlängen/ nach des S. H. D. Zachar. Stopii Abfatz. Von den unbeweglichen Feften und horizontal Sonnen=Uhr auff 57 2/3 Gr. Compaß. Das Kapitel enthält Stopius' Tabelle der Berechnungen der Zeiten (Gubert 1673: 45-60), denen die Auswertung ihrer Geltung erfolgt. Stopius hatte in Rostock studiert und dort 1569 seinen Doktortitel erlangt. Bekannt wurde er in Livland u.a. als Verfasser der Liefländifchen Oekonomie, die nur in Abschriften im Umlauf war und aus der Gubert 1645 Einiges in sein Stratagema oeconomicum übernahm, u. a. auch die von Stopius empfohlene Konstruktion der Sonnenuhr (Stradiņ̌̌ 2009: 123). In seinen letzten Lebensjahren hat sich Stopius der Landwirtschaft zugewendet. Man kann sich zwar vorstellen, dass Guberts Stratagema oeconomicum teilweise auch von Stopius' Manuskript inspiriert wurde. Dessen vollständige Auswertung ist aber mit Ausnahme der astronomischen Daten heute nicht mehr möglich, da sich weder das Manuskript noch seine Abschriften erhalten haben. Stradiņš nimmt an, dass Stopius' Werk und auch das Stratagema oeconomicum von Gubert für Livland angepasste Versionen der Publikationen Johann Colers gewesen sein dürften. In Guberts Stratagema oeconomicum aber wirke seiner Meinung nach auch die im damaligen Europa deutlich erkennbare Tendenz von Erkenntnissen antiker Autoren zur modernen empirischen Erfahrung und in das Werk seien auch Elemente aus den Natur- und Sozialwissenschaften integriert (vgl. Stradiņš 2009: 161). Im gesamten Text tritt Gubert auch als Pastor auf: Belege dafür findet man in erbaulichen Passagen, in zahlreichen Zitaten, oft aus der Bibel. Dadurch wird es möglich, auch den angedachten Adressaten des Buches detaillierter zu beschreiben - den gebildeten christlichen Leser, dem lateinische Zitatwörter, Phrasen und Sätze, zwar mit Übersetzungen, sowie der häufige Gebrauch von vielen in Antiqua gesetzten Fremdwörtern zugemutet werden können. 
Über Johann Hermann, dessen Lieffländifcher Landmann 1662 in Riga von Bessemesser aufgelegt wurde, ist weniger bekannt, auch wenn er laut Amelung (1884: 710) einer der „zwei namhafte[n] Vertreter“ der livländischen Hausväterliteratur und Nachfolger von Gubert gewesen sei. Gadebusch (1777: 65) vermutet, dass Johann Hermann - „ein erfahrner und glücklicher Landwirt in Livland“ - ein Anverwandter des aus Neidenburg stammenden Dichters Daniel Hermann (um 1543-1601) gewesen sein könnte. Hermanns Lieffländifcher Landmann wurde 1695 in Riga wiederholt bei Nöller aufgelegt. Eine Erwähnung in der Reihe der gedruckten Hausväterliteratur aus dem Zeitraum 1650-1750 fand Hermann neben Gubert bei Güntz (1897: 8). Der polnischen Übersetzung aus dem Jahre 1673 (wiederholte Auflage 1823) verdankt das Werk Hermanns seine Einbeziehung in den fachhistorischen Diskurs. So widmet Turzyński (2011: 8183) in seiner Untersuchung zur Buchhaltung an polnischen Gutshöfen des 17. Jahrhunderts seine Aufmerksamkeit u.a. auch dem Konzept Hermanns (poln. Jan Hermann). Weder in der germanistischen noch in der lettischen Linguistik ist dieses Werk näher analysiert worden, eher als Ausnahme kann seine Heranziehung in der Untersuchung des regionalen Wortschatzes von Uustalu erwähnt werden (vgl. Uustalu 1990).

Thematisch umfasst das Werk Hermanns mit Guberts Stratagema oeconomicum vergleichbare Bereiche - mit Ausnahme der astronomischen Daten. Als anschließendes Kapitel (Cap. XXXVIII-XL) enthält es Vorschriften für den Amtmann bei der Ausführung seines Amtes, u. a. auch darüber, wie der Amtmann seine Rechnungen führen und die Hofmutter ihr Amt verrichten sollen. Aus der Dem Erleuchten Hoch= und Wohlgebohrnen Grafen und Herrn [...] Magno Gabrieli de la Gardie gewidmeten Dedicatio wird in der Vorrede die Intention des Verfassers offenbar, die er mit seinem „Werklein“ verfolgt:

[...] ich möchte diefe meine auß der Erfahrenheit felbften gefchöpffte Wiffenfchafft/ der ich mich in fo vielen Jahren mit mehrerm Vortheil alß Schaden/ Gott Lob/ geübet/ von der Wirtfchafft zu Lande bey mir nicht erfterben/ fondern der pofterität durch den Drukk hinterlaffen [...]. (Hermann 1662: Vorrede)

\section{Einige Bemerkungen zum Inhalt der Texte}

Guberts Stratagema oeconomicum, dem das Vorwort des Druckers und die Vorrede des Verfassers vorangestellt sind, besteht aus drei Teilen, das Werk von Hermann umfasst außer der Dedicatio und Vorrede 40 Kapitel. Beide thematisch unterschiedlich strukturierten Werke zeigen viele inhaltliche Übereinstimmungen auf. Die Differenzen bestehen vor allem in der Heranziehung des 
Manuskripts von Stopius bei Gubert und der damit verbundenen astronomischen, auch astronomisch-technischen Abschnitte, wie z.B. über die Nutzung von Kompass und Herstellung der Sonnenuhr, ergänzt durch das auf die Wettervoraussagen orientierte livländische „Bauer=Prognoftikon“. Die Themenwahl ist in beiden Werken regional determiniert. Das betrifft die Beschreibung der Bodenbeschaffenheit und der landwirtschaftlichen Kulturen, der traditionellen Arbeiten in der Landwirtschaft, Jagd, Fischerei, Imkerei und auch einige handwerkliche Tätigkeiten, die dem Amt des Ackermanns oder Landmanns oblagen. Der Ackermann Guberts soll klar umrissene persönliche Voraussetzungen zur Ausübung des Amts mitbringen:

Ein Ackermann fol unter 60. Jahren alt feyn/ ein weifer verftändiger Mann/ der fürnemblich in wahrer Gottesfurcht (welche der Weißheit Anfang ift) lebet/ fleiffig beten/ die Seinigen/ wie auch die Arbeitsleute Abends und Morgens/ vor und nach dem Effen zum Gebät halten. (Gubert 1673: 1)

Gubert schildert außerdem viel ausführlicher solche Bereiche wie Viehzucht, Haltung von Haustieren und Geflügel sowie eine Reihe von wichtigen Tätigkeiten, wie z. B. Insektenbekämpfung, und auch handwerkliche Tätigkeiten, die für die Wirtschaftsführung unentbehrlich waren, wie z. B. Bauarbeiten. Bei Hermann beobachtet man dagegen eine stärkere Orientierung auf die praktische Ausübung der Tätigkeit des Amtmanns. Im XXXVIII. Kapitel beschreibt er die Fragen an den Bewerber vor dessen Anstellung seitens der Herrschaft:

Ob er nemlich ein Haußwirth/ und vorhin beym Land=Leben gewefen fei; Ob er weiß/ was eine richtige und redliche Rechnung fey/ demenach examinire man den Ambtmann von Akkerbau/ von der Saat=Zeit/ ja vom der gantzen Wirthfchafft [...] (Hermann 1662: 116)

Die Auswertung dieser Übereinstimmungen und Differenzen aus wirtschaftshistorischer Sicht, ergänzt durch das Material anderer landwirtschaftlicher Handbücher, könnte ein interessanter Beitrag zur livländischen, d. h. regionalen landwirtschaftlich orientierten Wirtschaftsgeschichte werden, da das Werk Hermanns als komplementär zu dem von Gubert betrachtet werden kann. Ein Vergleich beider Werke, den Amelung vornimmt, fällt aber zugunsten Guberts aus, dessen Schrift älter ist und:

[...] sie [beschäftigt] sich dafür bei ihrer grösseren Kürze und Einfachheit zum Glück auch nicht mit dem zum Theil völlig abergläubischen und wüsten Receptenkram des Joh. Hermann, sie erweist sich ferner als sehr freisinnig bei der Anwendung der damals unentbehrlichen Wetterdeutung und des vielen sonstigen Hocuspocus, der Zaubermittel u. a. m. (Amelung 1884: 710) 


\section{Informationsgewinnung und -präsentation: eigene Erfahrung und intertextuelle Bezüge}

Guberts Stratagema oeconomicum ist eine Verflechtung von Schilderungen eigener Praxis mit intertextuellen Bezügen. So erklärt Gubert z. B. den Kellerbau aus eigener Erfahrung, die dann anschließend als solche auch explizit hervorgehoben wird:

In der innerften Thür fetzet man ein Fenfter/ das wird allezeit offen gehalten/ ohne allein wenn es blitzet/ und wenn im Winter die kalten Nordwinde darauff ftoffen/ fo wird es zu gemacht. In folchem Keller ift mir in etlichen Jahren nichts gefroren. Ich kan auch Mertz= Bier bis auff Michael darinn gut behalten. (Gubert 1673: 101)

Einige Textpassagen sind rhetorisch in Frage-Antwort-Form strukturiert, die Gubert mit der Einleitung Hier fällt eine Frage für beginnt und in lapidar gehaltenem Stil mit Antwort weiterführt. Vgl. die Erörterung des Themas Winterweitzen=Saat:

Hier fällt eine Frage für: Obs Sünde fey/ den Roggen zu knicken/ daß der Weitzen beffer wachfen könne? Antwort: In der Vor=Riegen außzuziehen/ wenn man wegen der häuffigen Arbeit/ die in folcher Zeit fürfält/ darzu kommen könnte/ wäre beffer. Ich mache einen Unterfcheid zwifchen dem/ welchen man zur Saat/ und welchen man zur Taffel bedarff. (Gubert 1673: 150)

Ein anderes Verfahren der Textgestaltung sind die Zitate und darin folgt Gubert einem auch von Coler des Öfteren praktizierten Verfahren. Dem einleitenden Teil sind acht Zitate aus der Bibel vorangestellt, deren Schlüsselwörter - Substantive, Verben und Verbalphrasen - im Stratagema oeconomicum, bezogen auf verschiedene Themen, meist auch behandelt werden: pflanzet Bäume, fäet im Lande, ein Stükk Akkers, Vieh=hirten, drafch Weitzen, kaufft einen Akker, ein Kühehirt, Heerde, Akkermann. Als Illustrationen werden hier zwei Zitate angeführt: Genes. 21: Abraham pflantzet Bäume zu BerSeba (Genes. 21) und Zacharias nennet fich einen Akkermann (Zach. 13). Damit schafft Gubert einen fließenden Übergang zur Behandlung des im Titel seines Werkes angekündigten Themas.

Lateinische Zitate und deren deutsche Übersetzungen oder auch deutschsprachige Paraphrasen erfolgen meist mit Hinweis auf die Quelle, vgl. Plinius: Frons Domini plus prodeft in agro, qvam occipitium, Mit den Augen fchaffet der Herr dem Akker mehr Nutzen als mit dem Rükken (Gubert 1673: 3 f.) oder Cato: Res ruftica fic eft, fi unum fero feceris, omnia opera ferò facies. Mit dem Akkerbau ift es alfo befchaffen/ wo du ein Ding zu fpät thuft/fo gefchicht das andere auch zu fpat. (Gubert 1673: 4) 
Ähnlich wie bei Coler, nur in geringerem Umfang, finden sich bei Gubert gereimte Zitate oder sonstige gereimte Fragmente, vgl.:

\author{
Luth: Der Herre muß felbst fein der Knecht/ \\ Wil ers im Haufe haben recht/ \\ Die Fraue muß felbst fein die Magd/ \\ Wil fie im Haufe fchaffen Raht. (Gubert 1673: 7)
}

Durch die Zitate, die z. T. eine belehrende Funktion haben sollten, vollzieht sich gleichzeitig auch eine Lokalisierung der im Lateinischen bekannten Ausdrücke, wie z. B. im einleitenden Teil zum Thema Bemiftunge, vgl. Cato: Sterqvilinium magnum ftude ut habeas. Das ift: Lege fleiß an/ daß du viel Mift famleft. (Gubert 1673: 118 f.)

Der intertextuelle Bezug kann auch im Satz integriert werden, vgl. Die fschuppichten Fifche find gefunder/ als die fchleimmichten Fifche/ darumb Gott im alten Teftament den Juden Levit. 11. die Schleim=Fifche verbohten. (Gubert 1673: 127)

Die Ähnlichkeit der Zitierweise bei Coler und Gubert überrascht nicht, da die beiden Autoren auch der ausgeübte Beruf verbindet: Sowohl Coler als auch Gubert waren protestantische Pfarrer. Was aber als Anspruch auf Wissenschaftlichkeit interpretiert werden kann, das ist der Hinweis auf das vorher genannte Manuskript von Stopius. Auch auf andere das Fachgebiet betreffende Quellen gibt Gubert in seinem Text eindeutige Hinweise, z. B. Herr Colerus gibt in feinem Hauß=Buch dem Einfältigen nachfolgenden Bericht (Gubert 1673: 153) oder im Falle der Pferdeerkrankung: Wolfgangus fchreibet in Magia Naturali. Man fol ihme alßdenn/ wenn es fich eben ins Waffer legen wil/ einen neuen oder alten Pott auffm Kopff entzwey fchlagen [...] (Gubert 1673: 174) u. a. Vereinzelt erscheinen bei Gubert auch Hinweise auf die Autoritäten der Antike, wie im folgenden Beispiel: Defperatis Hippocrates verat ad hibere medicinam. Der berühmte Medicus Hippocrates läffet nicht zu/ daß man die Artzeney mißbrauchen fol bey denen/ welche die Hoffnung der Gefundheit verlohren. (Gubert 1673: $178 \mathrm{f}$.)

Gubert verwendet verschiedene wissenschaftliche Techniken. Einige Querverweise sorgen für die Kohäsion des Gesamttextes, vgl. Im Anfang diefes Monats ift die letzte Gerften/ aber die befte Buchweitzen=Saat/ biß an Viti. Befihe das Cap. von der Saatzeit. (Gubert 1673: 25) oder über die Mäusebekämpfung: Gute Mäufe=Katzen feyn beffer/ die verbieten ihnen leichtlich die Herberge. Befihe im 3. Theil das 7. Cap. (Gubert 1673: 109)

Hermann bezieht sich in seinem Werk kaum auf die Fachliteratur seiner Zeit, es geht ihm vor allem um die eigene Erfahrung, wie dies schon in der Vorrede unterstrichen wurde. Darin kann ein wesentlicher Unterschied zu Gu- 
bert festgestellt werden. Eine von Hermann in diesem Kontext verwendete idiomatische Wendung mit allgemeinem Hinweis kann eher als ornatus aufgefasst werden, vgl. Wie dann die Schrifft davon fpricht/ es foll kein Akker über fein Vermögen tragen. (Hermann 1662: 80)

\section{Adressatenbezogen verwendete grammatikalische Formen}

Die Kohäsion und Kohärenz des Gesamttextes wird von beiden Verfassern unterschiedlich erreicht. Der mit intertextuellen Bezügen reichlich ausgestattete Text von Gubert ist stilistisch ungleichmäßig, insbesondere im Bereich der Satzstilistik. Diese Verschiedenheit des Stils äußert sich im Wechsel von vollständigen und elliptischen Sätzen, wobei die Letzteren hin und wieder durch Item eingeführt werden können, wie dies eher für Urkunden charakteristisch ist, z. B. Zwei Tage nach dem vollen Monde fäet man Röttig. Item Rüben in den Bufchrödungen. (Gubert 1673: 25)

Eine Besonderheit der zuweilen lapidaren Formulierungsweise bei Gubert sind die Sätze mit Infinitiv. So finden sich bei der Beschreibung der Arbeiten im Monat März Sätze wie: Im Anfang diefes Monats/ das im Decemb. und Januario gefällete hinterftellige Bauholz außfchleppen. Im neuen Licht Egden bauen laffen. (Gubert 1673: 12) oder im September - Kapaunen machen. Den Elenden und wilden Gänfen nachftellen. Wacholderbern einfamlen. (Gubert 1673: 32) In diesen Beispielen lässt sich ein ökonomisches Sprachverhalten beobachten. Der Infinitiv erscheint hier, wie dies aus Anweisungen und Rezepten verschiedener Art bekannt ist, immer mit vorangestellten Determinanten.

Die Beschreibung der Saatzeit enthält eine detailliert geschriebene Passage, in der Infinitiv mit zu zum Ausdruck der Notwendigkeit verwendet wird:

die Sonne ist der Vater/ der Mond die Mutter/ der wachfenden Dingen/ derohalben die Zeit/ welche nach der Sonnen gerechnet wird/ und des Mondes Lauff/ fo viel müglich/ mit der Saat Außftreuung in acht zu nehmen. (Gubert 1673: 137 f.)

Der Beruf des Verfassers kommt im abschließenden Teil des Stratagema oeconomicum deutlich zum Ausdruck, er wird mit einem Gebet abgeschlossen (Gubert 1673: 257 f.), dem im Anhang geistliche Lieder deutsch und lettisch folgen. Betrachtet man das Werk von Gubert in seiner Gesamtheit, so wird deutlich, dass es trotz seiner Kohärenz, die durch thematischen Aufbau und fachsprachliche Formulierungen, intertextuelle Bezüge und erbauliche Einschübe realisiert wird, keine Einheitlichkeit in der Form aufweist - verschiedene Stilebenen und 
grammatische Mittel zur Gewährleistung der kommunikativen Zwecke hinterlassen gelegentlich einen eher heterogenen Eindruck. Nicht nachweisbar ist dabei der Einfluss des Manuskripts von Stopius. Ob Stopius' Text in unveränderter Form übernommen wurde und ob sich Gubert von weiteren Informationen inspirieren ließ, lässt sich ohne Vergleichsmöglichkeiten mit dem Ausgangstext nicht mehr nachweisen.

Hermanns Werk unterscheidet sich stellenweise in seiner sprachlichen Form stark vom Stratagema oeconomicum. In erster Linie ist dies an der Verwendung der Verbformen zu konstatieren. Zwar findet man einige Parallelen zu Gubert, z. B. in den Anleitungen in 2. Pers. Sg. Imp., wenn der Leser direkt aufgefordert wird, eine Handlung vorzunehmen, vgl. die Beschreibung der Insektenbekämpfung: Nimb eine gute Handvoll oder mehr Knoblauchs/ ftoß ihn klein/ lege felbigen in eine Tonne mit Waffer [...]. (Hermann 1662: 39)

Im Unterschied zu Gubert schreibt Hermann in langen ausführlich formulierten Sätzen und nutzt zur Aufforderung und Ausdruck der Notwendigkeit häufig Konj. Präs., vgl. Wann ein Haußman einen harten Bodem [...] gebe er acht/ daß fo balde das Land ein wenig/ als auff etwa 2. oder 3. Finger dikke tieff fich auffzuthun oder zu dauen beginnet/ er dafelbe alfo fort auffpflügen laffe [...]. (Hermann 1662: 7)

Vergleicht man die grammatikalischen Formen, die Lexik und Realisierung des Adressatenbezugs in beiden Texten, so fällt bei beiden Autoren eine differenzierte Bevorzugung grammatischer und lexikalischer Mittel auf. Das Werk von Gubert enthält diverse Formen, wie z.B. die in Urkunden charakteristischen Item-Einleitungen, elliptische Sätze mit Infinitiv in appellativer Funktion, die 2. Pers. Sg. Imp. oder 2. Pers. Sg. Ind. Hermann hingegen schreibt in einem eher homogenen Stil, seine Ausdrucksweise ist hypotaktisch und im Unterschied zu Gubert kann man in den anleitenden Passagen die präferierte Verwendung des Konj. Präs. Ind. beobachten.

\section{6 Übersetzungen aus dem Lateinischen}

Vom Leser des Stratagema oeconomicum wird erwartet, dass er gewohnt ist, Texte mit zahlreichen intertextuellen Bezügen und lateinischen Zitaten zu rezipieren. Dasselbe betrifft auch die vielen in Antiqua gesetzten Latinismen, die von beiden Verfassern verwendet werden. Als leserfreundliches Verfahren können bei Gubert die Übersetzungen der lateinischen Bezeichnungen, Phrasen oder sogar längeren Zitaten betrachtet werden, auf die der Verfasser an vielen Stellen den Leser auch aufmerksam macht. So findet man Formulierungen wie Die Naturkündiger nennen die Lufft Balsamum omnium corporum; Einen Balfam 
aller Cörper. (Gubert 1673: 91) und explizierende Hinzufügungen, wie z.B. ohne die Vordachung/ welche auf Lateinifch suggrundia genennet wird (Gubert 1673: 104 f.). Durch einzelne gereimte Zitate verliert der Text von Gubert seine Fachlichkeit, der Stil ist eher unterhaltend erbaulich, wie dies im folgendem Beispiel zu sehen ist:

Caro porcina, fine vino peior ovina:

Si tribuas vinum, fuerit cibus \& medicina.

Fleifch vom Schaf ist beffer als vom Schwein:

Artzney und Speiß/ ift Schweins Fleifch mit Wein. (Gubert 1673: 194)

Solche im Gesamttext verstreuten, gereimten Passagen kommen im Stratagema oeconomicum mehrfach vor, damit wird die Tradition, die man auch bei Coler beobachten kann, fortgesetzt. Die angeführten Beispiele, die z.T. eine unterhaltende Form haben, entsprechen eventuell den Erwartungen des Lesers. Das Nebeneinander von lateinischen Zitaten und deren Verdeutschungen signalisiert möglicherweise den Übergang zur deutschsprachigen Kultur. Hermanns Lieffländifcher Landman dagegen kann satzstilistisch eher als homogen betrachtet werden und auch auf der lexikalischen Ebene ist das Werk konsequent in der Präsentation der regional markierten lexikalischen Mittel, kombiniert gelegentlich mit Übersetzungen, wie dies im nächsten Kapitel gezeigt werden soll (s. Kap. 7.3.).

\section{Explikation der regionalen Zugehörigkeit der Texte}

\subsection{Zum Forschungsstand}

Die Regionalität der hier behandelten Quellen drückt sich verschieden aus. Neben der Themenwahl und den wiederholt explizit formulierten Hinweisen für den Leser soll im Kontext des Adressatenbezugs auch die regionale deutsche Sprache behandelt werden, die ein weiteres Indiz für die Orientierung an der livländischen Leserschaft ist. Das baltische Hochdeutsch als Folgesprache des davor dominierenden und im Laufe des 16. Jahrhunderts allmählich verdrängten Mittelniederdeutsch hatte zahlreiche Elemente des Letzteren aufgenommen, die, ergänzt durch einheimische lettische und estnische Bestandteile, die Spezifik der deutschbaltischen Lexik bildeten (vgl. Lele-Rozentāle 2016). Die niederdeutschen Bestandteile im Stratagema oeconomicum wurden seit den 80er Jahren des 20. Jahrhunderts von Uustalu in mehreren Aufsätzen behan- 
delt. Sie hat niederdeutsche Entlehnungen im Stratagema oeconomicum (Ausgabe von 1688) näher untersucht und einen starken niederdeutschen Einfluss festgestellt (Uustalu 1982). Das Werk von Gubert diente Uustalu neben Heinrich Gösekens Manuductio ad Linguam Oesthonicam (1660) auch als Quelle zur Untersuchung des Umlauts und dessen Abweichungen und Schwankungen, für die häufig der niederdeutsche Einfluss angenommen werden konnte (Uustalu 1984). Uustalu (1985: $81 \mathrm{f}$.) hat die Auffassung bestätigt, dass das baltische Deutsch ein niederdeutsches Substrat hat, und versuchte die Intensität des niederdeutschen Einflusses am Beispiel der baltischdeutschen Vokale der Stammsilbe, die in der betonten Silbe vom neuhochdeutschen Usus abweichen, an Hand der vorher erwähnten Quellen zu belegen. Der Text von Gubert wird von Uustalu als baltischdeutsch eingestuft und als häufigste Abweichung wird die baltischdeutsche Vokalkürze konstatiert, die an Stelle der nhd. Vokallänge in der Tonsilbe auftritt (Uustalu 1985: 87). Die Analyse der lexikalischen Elemente der regionalen Texte aus dem 17. Jahrhundert führen Uustalu zu der Schlussfolgerung, dass die landwirtschaftlichen Texte von Gubert und Hermann im Vergleich zu den Urkundentexten viele umgangssprachliche Elemente enthalten und dass die regionale Spezifik vor allem an niederdeutschen Elementen festzustellen sei (Uustalu 1990: 141). „Die landwirtschaftlichen Büchlein“, wie sie von Uustalu bezeichnet werden, „behandeln Probleme aus der dinglichen Umwelt, deshalb finden sich dort Lexeme, die wahrscheinlich aus der damaligen Umgangssprache herstammen“ (Uustalu 1990: 146).

Die von Uustalu durchgeführten Untersuchungen beziehen sich auf die phonetischen und lexikalischen Spezifika der Texte, vor allem des Stratagema oeconomicum, das Werk von Hermann ist an Einzelbeispielen analysiert. Für die Erforschung der frühen wissensvermittelnden Texte sind aber auch andere Faktoren von Bedeutung, wie z.B. die für die Entstehung eines fachsprachlichen Textes verwendeten Verfahren, die dessen Textualität ermöglichten, sowie auch die gezielte Regionalisierung bez. der Thematik, des sprachlichen Usus und der Erwartungen des Rezipienten. Aus diesen Überlegungen werden im Weiteren einige Hinweise auf die Region sowie auch einzelne Formen der Einführung regionaler lexikalischer Elemente behandelt.

\subsection{Explizite Hinweise auf die Region}

Hinweise auf die geographische Ausrichtung sind auffällige Merkmale. Die beiden Verfasser weisen auf Livland als Region der von ihnen beschriebenen Bodenbeschaffenheit und der territorial bedingten landwirtschaftlichen Tätigkeit hin. Schon die Titel beider Werke enthalten diese Information: Denen jungen 
ungeübten Akkersleuten in Liefland zum nöhtigen Unterrichte bei Gubert und Lieffländifcher Landman bei Hermann.

In Guberts Stratagema oeconomicum ist der regionale Bezug schon dadurch stärker ausgeprägt, dass er viele Daten von Stopius übernommen hatte, die in ihrer Verwendbarkeit geographisch begrenzt sind. Bei der Auflistung der Zeiten des Sonnenauf- und -niedergangs nach Stopius wird die Richtigkeit der angeführten Daten relativiert, vgl. ... fo wird fie doch in Churland/ im Baufchker Parallel etwas/ aber gar wenig/ fehlen/ in Littawen im Roffenifchen Parallel mehr [...] aber im Revalifchen Parallel mehr Unrichtigkeit haben [...] (Gubert 1673: 61). Es kann angenommen werden, dass diese Überlegungen von Stopius stammen, da die weiteren Ausführungen Kenntnis der Astronomie erfordern, dasselbe betrifft auch die Beschreibung der Ausfertigung der Sonnenuhr (vgl. dazu Gubert 1673: 66-69).

Die regionale Ausgerichtetheit erscheint unterschiedlich sprachlich formuliert durch das attributiv gebrauchte livländisch, vgl. aus der Beschreibung des Bauer-Prognostikons Die Lieffländifche Bauren nehmen in acht die Wochen vor Weihenachten/ und die Wochen nach Weihenachten (Gubert 1673: $77 \mathrm{f}$.$) oder$ auch in der Kapitelüberschrift bei Hermann, vgl. Cap. I. Vom Winter und deffen Lieffländifcher Arbeit (Hermann 1662: 1), sowie auch bei der Beschreibung von Personen bestimmter Stände, z. B. Daß die Lieffländifchen Land=Herren viel auff Wein wenden/ mit Verachtunge des edlen Gerften=weins/ darmit GOtt diß Land gefegnet/ kan ich nicht entfchuldigen (Gubert 1673: 227). Sonst tritt die regionale Fokussierung durch andere Mittel in Erscheinung, wie z. B. durch die hyperonyme Form Nordenländer, vgl. Aber im Vor=Jahr thut er [Nordwind, Dz. L.-R.] dem Roggen fchaden/ in diefen Nordenländern. (Gubert 1673: 159) Manchmal ist einfach von „diesem Lande“ geschrieben, dessen Identifizierung durch den Titel als gewährleistet gilt, vgl. In diefem Lande ift nicht zuträglich Holländifch Viehe zu halten (Gubert 1673: 184). Verwendet werden auch metonymische Bildungen, wie z. B. Die Leute in den Bier=Ländern leben eben fo lange/ als in den Wein= Ländern/ und überwinden jene mit Leibes=ftärkke (Gubert 1673: 228). Als weiterer Hinweis auf die Region kann der Vergleich der landwirtschaftlichen Arbeiten mit denen im benachbarten Estland aufgefasst werden, wie dies bei Hermann zu finden ist: [...] wil man aber mit Eichene[n] Zapffen oder Nägeln im Dröfchlande egge[n]/ ift beffer/ wie im Ehftlande gefchicht (Hermann 1662: 5) oder: In Ehftland/ wofelbften man wenig oder geringe Rödung fchläge/ machet man an ftat derfelben die Lohmes[...] (Hermann 1662: 25).

\subsection{Regionale Varianten mit dem Konnektor oder}

Die regionale Spezifik erscheint in beiden Texten in unterschiedlichen Formen. Als charakteristisch kann man Wortpaare anführen, deren ein Bestandteil ein 
baltischdeutsches Äquivalent zum hochdeutschen Wort ist, verbunden mit dem Konnektor oder. Diese Art zu formulieren wird vor allem von Hermann bevorzugt. Es handelt sich in vielen Fällen um lokal verbreitete lettische, vereinzelt auch estnische Lehnwörter oder um regionale, vorwiegend aus dem Niederdeutschen entlehnte und in Livland bekannte Fachwörter, die zur Entstehung der regionalen Varietät des baltischen Deutsch beigetragen haben. Mit dem Konnektor oder nimmt Hermann in diesen Fällen eine sprachlich determinierte Wahl vor. Was seine Intention betrifft, so könnte man sich vorstellen, dass dadurch als Landmann auch derjenige, der des regionalen Deutschen nicht mächtig war, in die Lage versetzt werden sollte, die Aufsicht über die landwirtschaftlichen Arbeiten in Livland zu übernehmen. Es geht hier dementsprechend um Informieren und Belehren. Der Konnektor oder wird auch in anderen Funktionen verwendet. Man kann zahlreiche inhaltlich determinierte Variationsmöglichkeiten beobachten, wie z. B. vom Regen oder Schnee (Hermann 1662: 56), im zunehmenden= oder vollen Mond (18), voller Äfte oder Zweigen fein (53), zwey oder drey Körnlein (28) u.a. Diese Fälle werden hier nicht behandelt. Weiter wird nur die einleitend genannte regional markierte Gruppe der parallel gebrauchten Fachwörter mit dem Konnektor oder näher betrachtet und mit den Einträgen in den Sammlungen regionalspezifischer Wörter abgeglichen. Ihr Vorhandensein in den regionalen Wortschatzsammlungen gilt als Nachweis des vom hochdeutschen Usus abweichenden Gebrauchs. Bevorzugt wird hier zum Vergleich das Idiotikon der deutfchen Sprache in Lief- und Ehftland (1795) von Hupel ${ }^{5}$ herangezogen, worin gelegentlich auch Verweise auf Gustav Bergmanns Sammlung Livländifcher Provinzialwörter (Salisburg 1785) enthalten sind.

\subsubsection{Niederdeutsche Elemente}

\section{Modder oder Schlamm}

Im Wortpaar Modder oder Schlamm (Hermann 1662: 90) ist Modder die niederdeutsche Entsprechung zu Schlamm, vgl. mnd. mod[d]e, mudde ,Schlamm (in Gräben)‘. (Schiller \& Lübben 1877, III: 106)

\section{Molkk(e) oder Waddak}

Das Wortpaar Molkk oder Waddak (Hermann 1662: 62), Molkke oder Waddak (140) enthält ein regional verbreitetes niederdeutsches Wort, vgl. mnd. wadde-

5 August Wilhelm Hupel (1737-1819), Pastor und Literat, geboren im Herzogtum Weimar, kam im Alter von 20 Jahren ins Baltikum. Sein Interesse galt neben verschiedenen anderen Bereichen auch der regionalen Variante der deutschen Sprache, insbesondere deren Lexik. 
ke, wadike, watke ,Käsewasser; was von der Milch zurückbleibt, nachdem Butter oder Käse daraus gemacht wird‘ (Schiller \& Lübben 1880, V: 571).

\section{Stangen oder Ricker}

Stangen oder Ricker von Thannen (Hermann 1662: 6) ein paar Stangen oder Rükker (41), auff Stangen oder Rükker hengen (43), von ftarkken Rikkern oder Stangen (55), eine Scheune von Rikkern oder Stangen (58).

Hupel (1795: 192) lemmatisiert das regional gebrauchte Fachwort Ricker in seinem Idiotikon der deutfchen Sprache in Lief- und Ehftland und gibt dazu eine Erklärung: Es bedeute "bey dem rigischen Holzhandel eine Stange, Latte, auch einen dünnen Balken. Einige verstehen dadurch kleine Latten. S. auch Rücker“. Auch Bergmann (1817: 216) führt es als eines der regionalen Wörter an: Ricke, Ricker ,dünne Stangen, daran noch die Rinde ist'. Laut Schiller \& Lübben bedeutet das mnd. rick, reck ,eine lange dünne Stange‘ (Schiller \& Lübben 1877, III: 475).

\section{wehden oder außjähten}

Im Wortpaar wehden oder außjähten (Hermann 1662: 88) erscheint das aus dem Mittelniederdeutschen stammende wehden als regionales Synonym für ausjäten, vgl. mnd. weden ,Unkraut ausraufen“ (Schüller \& Lübben 1880: 645). In seinem Idiotikon führt es auch Hupel (1795: 260) an.

\section{trucknen oder dorren}

Das Wortpaar trucknen oder dorren (Hermann 1662: 7) enthält die niederdeutsche Entlehnung dorren, die auf das mnd. dorre ,trocken' zurückgeht (Schiller \& Lübben 1875, I: 553). Das Wort fehlt im Wörterbuch von Hupel.

Durch oder werden auch regionale parallele Varianten verbunden, die als stilistisch markiert gelten, vgl. eine Handvoll verbunden Hopffen oder einen Knuppen von Hopffen (Hermann 1662: 77). Laut Hupel soll der Knup statt Knoten als pöbelhaft gelten und nach Bergmann bedeute es auch, ein Bündel oder Päckchen' (Hupel 1795: 120).

In einigen Fällen wird auf den regional bedingten Gebrauch explizit im Text hingewiesen, wie z. B. durch die Anmerkung „wie bei uns genannt“. In solchen Fällen handelt es sich um vollständige Übereinstimmungen, vgl. einen harten Bodem/ oder wie bei uns genandt/ Dröfche (Hermann 1662: 7). Laut Hupel (1795: 52) bezeichnet man als Drefche Ackerland, ,wenn es lange unbearbeitet gelegen hat und daher ganz begraset ist.“ Es geht auf das mnd. dresch, drisch zurück, ,[...] eine wenig fruchtbare, unbebaute, als Trift benutzte Strecke, die nur spärlich mit Gras bewachsen ist' (Schiller \& Lübben 1875, I: 573). 
Manchmal trifft man mit oder verknüpfte Parallelformen, bei deren einem Element eine niederdeutsche Lautung enthalten ist, wie z. B. der Vorwinter gehet oder dauet weg (Hermann 1662: 91), vgl. mnd. douwen. Nach Gutzeit (1864: 179) seien das Verb dauen und das Substantiv Dauwetter statt tauen und Tauwetter noch in den ersten Jahrzehnten des 19. Jahrhunderts in Gebrauch gewesen.

\subsubsection{Regional gebrauchte deutsche Synonyme}

Einige Konstruktionen enthalten deutsche Synonyme, die in den regionalen Wortschatzsammlungen mit dem Vermerk selten angeführt werden. Dazu gehören auch die Wortpaare mit Vorjahr, vgl. im Vorjahre oder in der letzten Winterzeit (Hermann 1662: 5), im Frühling oder Vorjahr (21). Hupel (1795: 254) erwähnt beim Stichwort Vorjahr, dass es statt Frühling erscheint und selten gebraucht wird.

\subsubsection{Parallelformen mit lettischen und estnischen Entsprechungen}

Eindeutige Übereinstimmungen sind die Konstruktionen mit den nichtdeutschen Entsprechungen. Das sind meist lettische und in Einzelfällen auch estnische Äquivalente zu den deutschen Realienbezeichnungen. Im Weiteren folgen einige Beispiele, von denen auch ein Teil zur regionalen deutschen Sprache im Baltikum gehörte.

\section{Buchweitzen oder Gricke}

Buchweitzen oder Gricke (Hermann 1662: 11), Buchweytzen oder Grikken (14), 3. Loff Buchweitzen oder Grikken (142). Interessanterweise ist Hermann in seinen Ausführungen konsequent beim Gebrauch des Wortpaars und dies trotz dessen wiederholter Erwähnung im Text. Als landesspezifische Bezeichnung wird Gricke mit Hinweis auf lettische Herkunft auch im Idiotikon von Hupel angeführt (Hupel 1795: 82). ${ }^{6}$ Polanska (2002: 308) vermutet den Gebrauch der lettischen Bezeichnung ausschließlich „bei den unteren Schichten der Deutschen“ und begründet diese Annahme damit, dass Gubert in seinem Werk konsequent nur die Bezeichnung Buchweitzen verwendet.

6 Ausführliche Information und Literaturhinweise zu dieser und weiteren Entlehnungen, die im baltischen Deutsch als sichere Entlehnungen aus dem Lettischen gelten s. bei Polanska (2002: 195-316). 


\section{Fußgänger oder Oterneken}

Fußgänger oder Oterneken (Hermann 1662: 34) ist eine weitere Konstruktion mit lettischer Bezeichnung. Gutzeit (1864: 303) führt in seinem Wörterbuch Fußgänger für ,Fußarbeiter“ an und erklärt ihn als „Bauer, der zu Handdiensten gestellt wird, was gew. von Georg bis Michael geschieht.“ Bei Hupel (1795: 166) ist die lettische Entlehnung Oternek, ein Fronarbeiter zu Fuß, ein Handfröhner oder Handarbeiter‘ (vgl. auch Polanska 2002: 248).

\section{Speicher oder Klehte}

Die lettische regionale Entsprechung zu Speicher - Klehte - gehört zum festen Bestandteil des baltischdeutschen Wortschatzes. Hermann verwendet sie synonymisch zum Speicher, vgl. aus dem Speicher oder Klehten (Hermann 1662: 125). Auch bei Gubert findet sich eine Konstruktion mit dieser Entlehnung Kleht oder Kornhauß (Gubert 1673: 104), wobei Kleht als das Kleht erscheint. Hupel (1795: 115) vermutet bei der Bezeichnung die Kleete eine Entlehnung aus dem Lettischen und zählt als Entsprechungen Vorratshaus, Speicher, Magazin auf, wobei auch Komposita genannt werden, vgl. Kornkleete für Kornkammer oder Kornspeicher; Mehlkleete für Mehlmagazin und Handkleete für Vorrathskammer ,in welcher allerlei Bedürfniffe, Hülfenfrüchte u. d. g. aufbewahrt werden“ und Leihekleete als Bezeichnung für Magazin, ,aus welchem die Gebietsbauer ihren Vorfchuß bekommen u.d.g. m. "Hingewiesen wird dabei auf die damalige Ansicht, dass dieses Wort auch aus dem Russischen hergeleitet werden könnte, „bald von Klet oder Kljet die Wohnung, Hütte, bald von Kletki die Honigzellen” (Hupel 1795: 115). Dieselbe Unsicherheit bez. der Herkunft ist in der Fachliteratur bis heute erhalten geblieben (vgl. Karulis 1992: 403-404).

\section{der Kubias oder Staroft}

Unter den einheimischen Sprachen dominiert in den festgestellten Konstruktionen als Äquivalent vorwiegend das Lettische, in Einzelfällen finden sich auch estnische Entsprechungen, vgl. der Kubias oder Staroft (Hermann 1662: 124), wo die Bezeichnung Kubjas ,Auffeher bey Frohnarbeiten in ehftnifchen Diftrikten' dem Estnischen entnommen und entsprechend im estnischen Teil auch verbreitet ist (Hupel 1795: 130). Der auf das Russische zurückgehende Staroft oder Staraft hingegen wurde im lettischen Teil als ,Bauerauffeher bey den Frohndienften in Lettland auch in einigen von Ruffen bewohnten Dörfern gebraucht (Hupel 1795: 226-227).

\section{Schoppen oder Kuyen; Schoppen oder Gubben (?)}

Estnischer Herkunft ist auch das Substantiv Kuyen in der Konstruktion in den Schoppen oder Kuyen (Hermann 1662: 141). Laut Hupel (1795: 131) ist das estni- 
sche Kui oder Kuje ,ein großer kegelförmiger Haufen z. B. Stroh, Heu, Korn‘. Im lettischen Teil Livlands wird die Bezeichnung Gubbe gebraucht, deren Semantik sich etwas von dem für das estnische Wort Festgestellten unterscheidet, auch wenn angenommen werden kann, dass Gubbe im Wortpaar auff die Schoppen oder Gubben (Hermann 1662: 119) als Äquivalent zu Schoppen gebraucht ist. Vgl. aber die Bedeutungserklärung im Wörterbuch von Hupel: Das aus dem Letischen entlehnte Substantiv Gubbe sei ,ein kleiner $\mathrm{Heu}=$ oder Korn=Haufen unter freien Himmel‘. (Hupel 1795: 83-84). Aus dem Kontext geht nicht hervor, ob mit dem deutschen Schoppen verschiedene Größen des Heuoder Kornhaufens bezeichnet werden konnten, da die estnische und lettische Bezeichnungen unterschiedliche Größen benannten, außerdem war die lettische Entlehnung auch im estnischen Teil verbreitet, vgl. Gubbe, Kubbe (mehr bei Polanska 2002: 309).

\subsubsection{Explizite Hinweise auf den regionalen Sprachgebrauch}

In vielen anderen Fällen gibt es explizite Hinweise auf die lettische oder andere in der deutschbaltischen Umgangssprache verbreitete Ausdrucksweise, vgl. wie es die Bauren nennen in: die Forche/ oder Byrzezems wie es die Bauren nennen (Hermann 1662: 17); fonsten genannt in: die gelbe Blumen oder Donnerkraut/ fonften Perkaunfaal genannt (88); wie bey uns genandt in: mit Sicheln/ oder wie bey uns genandt/ Sirpen zu mähen (46) oder ohne konkrete Angabe, nur auf die Umgangssprache hinweisend wie man faget in: Auch ifts nicht gut umb die Zeit zu fäen/ in welcher der Mond hökerig/ oder wie man faget/ unterwerts ftehet/ oder auch einen Pukkel auf dem Rükken hat (93). Hier geht es eher um eine Paraphrase, die im Werk von Hermann oft vorkommt und die weiter unten kurz behandelt wird.

\subsubsection{Paraphrasen}

Das Werk Hermanns enthält zahlreiche durch oder, seltener durch und verbundene Paraphrasen, die hier weiter angeführt werden: im letzten Schlitten=Wege oder Bahn (Hermann 1662: 4); ein wenig oder etwas Graß (10); In Ehftland/ wofelbften man wenig oder geringe Rödung fchläget (25); dann der Mift bleibet defto beffer mit der Erden vermifchet und verwächfelt (32); die Garbe oder Bunde (40); fich nicht gar auff einander fakke oder fenke (42); was die Schweine umbgewühlet oder geweltzet haben (58); außkeimen oder außfchlagen (65); Wann man Maltz nur etwas oder wenig keime[n] läft (67); Hochzeit=Bier brauen oder 
kochen (69); Hopffen/ welcher grün oder unzeitig gerupfft wird (72); denn er fich nicht wohl handtieren oder handhaben läft (83); fein glatt oder eben machen (90); in ein Land oder Akker (93); daß fie fich ausgedehnet oder gerekket halten (106).

Einige der von Hermann gebrauchten Paraphrasen deuten auf den Versuch hin, erst zu explizieren, bevor ein Fachwort angeführt wird, vgl. Von Netz und Garnen machen oder ftrikken (Hermann 1662: 103), wann grob Gahren allein gearbeitet oder geftrikket/ wird (104); einen Mahßftokk von 5. der Netze Augen oder Mafchen (106); die Augen oder Mafchen (107); deren Auge[n] oder Mafchen (108). Hierher gehört auch das Wortpaar beffern Nutzen oder Profit ${ }^{7}$ (9).

Gubert ist in seinem Text viel sparsamer mit synonymischer Verwendung von Fachwortschatz und meist handelt es sich dabei um Realienbezeichnungen, die semantisch nahestehende, aber unterschiedliche Denotate benennen. Eine Ausnahme bildet die schon erwähnte Phrase Kleht oder Kornhauß. (Gubert 1673: 104)

\section{Livländisches Fachwissen am Beispiel der Klassifikationen und Bezeichnungen von Bodenarten}

Die von Gubert vorgenommene Beschreibung des Bodens (auch Ackers oder Erde) kann als eine Weiterentwicklung der Bodenwissenschaft im Vergleich zu Coler betrachtet werden, dessen Beschreibung des Ackers in erster Linie auf die Merkmale fokussiert war: „Wobey ein guter Acker zu erkennen ift“. (Coler 1645: 108) Der Beitrag Guberts gehört zum traditionellen Bestandteil der baltischen Bodenkunde. So verweisen Reintam et al. (2005: 121) darauf, dass Gubert neben „Braun Land, Grau Acker and Grau Land“ auch drei Arten der schwarzen Erde beschreibt, „Schwarze Erde, Schwarz Acker and Schwarz Land.“

Die Einteilung der Bodenarten (der Lande bei Gubert, der Äkkere bei Hermann) unterscheidet sich in beiden Werken. Gubert schreibt Vom Unterfchiede der Lande (Gubert 1673: 116-118) und führt unter Mift=̈̈kker folgende zehn Lande (auch Erden und Äcker) an, die bez. ihres Nutzens für die Landwirtschaft von Gubert in seinem Stratagema oeconomicum auch eingehender charakterisiert sind:

1. fchwarze Erde [...] die werden von den Bauren maife femme genennet (116 f.);

2. Grau Akker/ welcher zum Grunde Leem hat (117);

7 Das Fremdwort Profit ist nicht in Antiqua gesetzt. 
3. Schwarz Land mit vielen kleinen Kiefelfteinen vermenget (117);

4. Schwarz Akker/ der zum Grunde Klint oder Kalkftein hat (117);

5. Akker mit vielen Kalkfteinen vermenget (117);

6. Braun Land/ welches zum theil Leem/ zum theil Sand zum Grunde hat (117);

7. Grau Land (117);

8. Werder $=$ Lande (117 f.);

9. Leem Lande von rohtem oder grauen Leem (118);

10. Heide=Lande (118).

Hermann bietet eine zum Teil divergierende Klassifikation der sechferley $\ddot{A} k$ kere (79-83) an, deren Kurzbeschreibungen hier angeführt werden sollen:

1. Der erfte und befte Akker ist von grauer Erden mit kleinem Grande/ als Staub=Perlen vermifchet/ der Grund unten mit rohtem Leyme fundiret (79);

2. Der ander Akker ift schwartzer Erden mit einem leimichten Grunde (80);

3. Der dritte Akker ift grauer und weißlichter Erden oder Kley (80);

4. Der vierdte Akker ift von grauen Sande/ darunter ein gelber Leym=Grund ift (81);

5. Der fünffte Akker ift vom rothen Sande (82);

6. Der sechfte Akker ift von rohtem Leyme (83).

Die Klassifikationen, fachsprachlichen Formulierungen und Fachwörter im Bereich Bodenkunde unterscheiden sich bei beiden Autoren. Unterschiedlich ist auch die Grundeinheit der Klassifikation: Gubert führt Erde, Acker und Land an, Hermann dagegen konzentriert sich in erster Linie auf den Acker. Inwieweit hier originäre Klassifikationen und Bezeichnungen gebraucht werden, sollte in diatopisch orientierten vergleichenden Quellenstudien geklärt werden. Unter den Fachwörtern hat das Kompositum Schwarzerde eine besondere Stellung, da es oft in der Fachliteratur zur Bodenkunde oder Bodenwissenschaft mit dem Namen Guberts in Verbindung gebracht wird. Laut Reintam ${ }^{8}$ kann die weltweite Verwendung des Fachworts auf Gubert zurückgeführt werden. Guberts Stratagema oeconomicum wurde 1747 von Lomonossow ins Russische übersetzt und durch die Lehnübersetzung der deutschen Bezeichnung Schwarzerde ins Russische ist das Fachwort Chernozem später von W. W. Dokutschajew in dessen Bodenklassifikation übernommen worden. Reintam et al. (2005: 121) weisen auf die Rolle der Übersetzung hin: „As a result of translation by Lomonosov (1747), the first of the three [die anderen zwei sind Schwarz Acker und Schwarz Land; Dz. L.-R.] came to enrich not only Russian but also

8 Zit. nach Alterman et al. (2005: 726); vgl. auch Blume (2009: 435); Vysloužilová et al. (2016: 85). 
world soil classifications under the term Chernozem. It means that contemporary Chernozem as a type of steppe, forest steppe, and prairie originates from Livonian (Southern Estonia and Northern Latvia) oak stands." Alterman et al. (2005: 726) nehmen mit Hinweis auf Reintam an, dass „[i]t is quite possible, that the terms Tschernosjom or Tschernosem or Chernozem comprise the oldest derivations for a soil type.“

Für die Entwicklung der frühen agrarwissenschaftlichen Fachlexik sowie für ihre Vermittlung ist es wichtig, nicht nur die Termini, sondern auch die dazu gehörende Umgebung als Konstruktionen oder feste Wortverbindungen kollokativer Natur festzustellen. Hier soll zuerst erwähnt werden, dass die den Boden bezeichnenden Fachwörter in den untersuchten Werken allem Anschein nach nicht selten synonymisch verwendet wurden, wenn auch gewisse Differenzen in deren Distribution festzustellen sind. Eine weitere detaillierte Behandlung der Fachlexik, angefangen mit dem kontextuellen Gebrauch von Erde, Land, Acker und Boden in Verbindung mit den vorgeschlagenen Klassifikationen, erfordert vergleichende Studien mit Einbeziehung einer umfangreichen Quellenbasis.

\section{Fazit und Ausblick}

Das Stratagema oeconomicum von Gubert ist in mehrfacher Hinsicht ein Beispiel allmählicher Entfernung von der Tradition, die von Coler konstituiert wurde. Somit geht die darin beobachtete sprachliche Entwicklung mit den von Wissenschaftshistorikern beobachteten inhaltlichen Neuerungen einher (s. Kap. 2 in diesem Beitrag).

Auch wenn die Zeitspanne zwischen der ersten Ausgabe des Stratagema oeconomicum von Gubert (1745), die 1773 zum dritten Mal aufgelegt wurde, und der Veröffentlichung des Lieffländifchen Landmans von Hermann (1662) nur 17 Jahre umfasst und auch die behandelten Themen vergleichbar sind, so lassen sich bei beiden Verfassern unterschiedliche Schreibtechniken und -stile beobachten. Wenn Gubert mit Ausnahme der von Stopius übernommenen Fragmente oft eher die Tradition von Coler fortsetzt, so lässt sich bei Hermann eine neue Tradition und Schreibtechnik beobachten: Sein Werk enthält kaum intertextuelle Bezüge, dem Leser wird viel komplizierterer Satzbau zugemutet und gleichzeitig entfaltet er eine intensive Beschäftigung mit der Fachlexik, deren regionale Varianten, präsentiert durch Konstruktionen mit dem Konnektor oder, sich besonders für eingewanderte Landwirte als zweckmäßig erweisen konnten. Ob das Vorgehen des Letzteren bei der Einführung der Regionalismen und Synonyme nur ein livländisches oder individuelles Phänomen darstellt 
oder einer bislang nicht identifizierten Tradition entstammt, soll weiteren vergleichenden wissenschafts- und fachsprachlichen Studien vorbehalten bleiben. Auch die grammatikalischen Voraussetzungen zur Entstehung der stilistischen Heterogenität wie in Guberts Stratagema oeconomicum oder der Homogenität wie in Hermanns Lieffländifchen Landmann könnten auf einer erweiterten Quellenbasis weiter untersucht werden.

Die wissenschaftlich und praktisch angelegten historischen Agrarbücher, die oft wiederholte Auflagen erlebten, wurden nicht selten übersetzt (vgl. dazu Hahn 2014), wie dies auch mit den hier behandelten Werken geschah. Da die Übersetzungen aber ihre Anwendbarkeit nur durch Anpassung an die lokalen Gegebenheiten erreichen konnten, ist die Analyse dieser Texte auch aus übersetzungstheoretischer Sicht von Interesse. Für die beiden hier behandelten Texte kommen die russische und die polnische Übersetzungen in Frage. Dass das Übersetzen auch innerhalb eines Textes relevant war, wurde an der Vielfalt der eingesetzten Verfahren in den Texten von Gubert und Hermann sichtbar, auch wenn es dabei nur um Elemente, nicht um Gesamttexte ging.

Die hier behandelten Aspekte umfassen nur einen Teil der festgestellten Spezifika, auch einige Beobachtungen konnten in diesem Beitrag nicht berücksichtigt werden, da für ihre Interpretation eine breit angelegte vergleichende Studie erforderlich wäre. Als weitere Perspektive könnte man die Entstehung der agrarwissenschaftlichen Fachlexik in diatopischer und diachronischer Perspektive betrachten, deren Schwerpunkte auch in der Feststellung der wissenschaftlichen Kontakte und Einflüsse liegen könnten.

\section{Quellen}

Gubert, Salomon (1673): Stratagema oeconomicum oder Akker=Student/ Denen jungen ungeübten Akkersleuten in Liefland zum nöhtigen Unterrichte/ vermittelft vieljährigen Obfervationibus, auch fürnehmer Philofophorum Placitis dargeftellet/ Letzt=vermehrete Edition. Riga: Bessemesser.

Hermann, Johann von Neidenburg (1662): Lieffländifcher Landman. Riga: Bessemesser.

\section{Sekundärliteratur}

Adelung, Johann Christoph (1787): Fortfetzung und Ergänzungen zu Chriftian Gottlieb Jöchers allgemeinem Gelehrten=Lexicon, worinn die Schrifttteller aller Stände nach ihren vornehmften Lebensumftänden und Schriften befchrieben werden. Leipzig: Gleditsch.

Altermann, Manfred et al. (2005): Chernozem-Soil of the Year 2005. In: Journal of Plant Nutrition and Soil Science 168 (6), 725-740. http://dx.doi.org/10.1002/jpln.200521814 (06. 10. 2018). 
Amelung, F. (1884): Salamo Gubert, der Vater der livländischen Landbauwissenschaft. In: Baltische Monatsschrift 31, 709-727.

Amelung, F. (1885): Ein Vademecum livländischer Landwirthschaft vor zweihundert Jahren. In: Baltische Monatsschrift 32, 147-164.

Andronova, Everita (2006): Latviešu valodas teksti Zālamana Guberta Stratagema oeconomicum 1645. gada pirmizdevumā un citos 17.gs. izdevumos (Die lettischen Texte in der ersten (1654) und weiteren Auflagen des 17. Jahrhunderts des Stratagema oeconomicum von Salomon Gubert). Vortrag auf der 11. wissenschaftlichen Konferenz Vārds un tā pētī̌anas aspekti. 30. 11.-1. 12. 2006, Liepāja.

Bergmann, Liborius (1817): Glossarium. In: Ders. (Hrsg.), Fragment einer Urkunde der ältesten Livländischen Geschichte in Versen. Riga: Wilhelm Ferdinand Häcker, 210-220.

Blume, Hans-Peter (2009): Soils and Soil. Pioneers on Stamps. In: Edward R. Landa \& Christian Feller (Hrsg.), Soil and Culture. Dordrecht u. a.: Springer, 431-438.

Coler, Johann (1645): Oeconomiae. Das Vierdte Buch. Vom Ackerbau. Georgia oder Georgica genandt. In: Johann Coler, Oeconomia. Ruralis et Domestica. Mainz: Hayln, 89-148.

Gadebusch, Friederich Konrad (1777): Livländische Bibliothek nach alphabetischer Ordnung. Bd. I-Il. Riga: Hartknoch.

Güntz, Max (1897): Handbuch der Landwirtschaftlichen Litteratur. I. Teil. Bis ca. 1750. Mit biographischen Notizen und 6 Bildnissen von Autoren. Auf Grund von Bibliotheksstudien. Leipzig: Voigt.

Gutzeit,Woldemar von (1864): Wörterschatz der deutschen Sprache Livlands. I. Bd. Riga: Kymmel.

Hahn, Philip (2014): Nutz, Pflicht und Vergnügen: Umweltwahrnehmungen im europäischen Landwirtschaftsschrifttum des 16. und 17. Jahrhunderts. In: Manfred Jakubowski-Tiessen (Hrsg.), Von Amtsgärten und Vogelkojen. Beiträge zum Göttinger Umwelthistorischen Kolloquium 2011-2012. Göttingen: Universitätsverlag, 49-65.

Hupel, August Wilhelm (1795): Idiotikon der deutschen Sprache in Lief- und Ehstland. Nebst eingestreueten Winken für Liebhaber. Riga: Hartknoch.

Karulis, Konstantīns (1992): Latviešu etimologiijas vārdnīca divos sējumos. Riga: Avots.

Lele-Rozentāle, Dzintra (2016): Vom Niederdeutschen zum Hochdeutschen: historischer Einblick in die Geschichte des baltischen Deutsch. In: Ineta Balode \& Dzintra LeleRozentāle, unter Mitwirkung von Manfred von Boetticher \& Reet Bender, Deutsch im Baltikum. Eine annotierte Forschungsbibliographie (Fremdsprachen in Geschichte und Gegenwart. Bd. 17). Wiesbaden: Harrassowitz, 37-63.

Polanska, Ineta (2002): Zum Einfluss des Lettischen auf das Deutsche im Baltikum. Inaugural-Dissertation in der Fakultät Sprach- und Literaturwissenschaften der OttoFriedrich-Universität Bamberg. Bamberg.

Recke, Johann Friedrich von \& Karl Eduard Napiersky (1829): Allgemeines Schriftstellerund Gelehrten-Lexikon der Provinzen Livland, Ehstland und Kurland. Zweiter Band. G-K. Mitau: Steffenhagen und Sohn.

Reintam, Loit, Igna Rooma, Ain Kull \& Raimo Kõlli (2005): Soil Information and its Application in Estonia. In: Robert J. A. Jones \& Beata Houšková \& Peter Bullock \& Luca Montanarella (Hrsg.), Soil Resources of Europe second edition. European Soil Bureau Research Report No. 9. Luxembourg: Office for Official Publications of the European Communities, 121132. http://library.wur.nl/isric/fulltext/isricu_t4d6d164f_001.pdf (06.10. 2018).

Schiller, Karl \& August Lübben (1875-1881): Mittelniederdeutsches Wörterbuch. Bd. I-VI. Bremen: Kühtmann u. a. 
Stradiṇ̌̌, Jānis (2009): Zinātnes un augstskolu sākotne Latvijā (The Beginning of Science and Higher Education in Latvia). Riga: Latvijas vēstures institūta apgāds.

Turzyński, Mikołaj (2011): Bookkeeping in Manor Farms of Polish Gentri in 17th Century. In: Eurasian Journal of Business and Economics 4 (8), 71-86.

Uustalu, Koidu (1982): Niederdeutsche Elemente in Baltisch-Deutsch des XVII. Jh. In: Kõrvutava ja rakenduslingvistika küsimusi. Linguistica 15 (Tartu Riikliku Ülikooli Toimetised. Bd. 619). Tartu, 151-158.

Uustalu, Koidu (1984): Sprachgeschichtliche Bemerkungen zur deutschen Sprache in Estland und Lettland im XVII. Jh. - zum Umlaut. In: Linguistica 17 (Tartu Riikliku Ülikooli Toimetised. Bd. 684). Tartu, 130-139.

Uustalu, Koidu (1985): Niederdeutsches Substrat im baltischdeutschen Lautbestand des XVII. Jh. - Vokalismus. In: Linguistica 18 (Tartu Riikliku Ülikooli Toimetised. Bd. 713). Tartu, 81-89.

Uustalu, Koidu (1990): Baltischdeutsche Sprachvarianz in Estland und Lettland im XVII. Jh. In: Uurimusi germaani keelte sõnavara ja grammatika alalt. Linguistica 22. Tartu, 140-147.

Vysloužilová, Barbora, Damien Ertlen, Dominique Schwartz \& Luděk Šefrna (2016):

Chernozem. From Concept to Classification: A Review. In: Acta Universitatis Carolinae. Geographica. Univerzita Karlova 51 (1), 85-95. 